\title{
Um ambiente para autoria e realização de aplicações educacionais com realidade aumentada
}

\author{
Thiago Z. Fraga ${ }^{1}$, Crediné Silva de Menezes ${ }^{1,2}$ \\ ${ }^{1}$ Programa de Pós-Graduação em Informática - Universidade Federal do Espírito Santo \\ (UFES) \\ ${ }^{2}$ Faculdade de Educação - Universidade Federal do Rio Grande do Sul (UFRGS) \\ thiagozamborlini@gmail.com, credine@gmail.com
}

\begin{abstract}
The augmented reality allows the student to visualize an idea, previously only mentalized from the teacher's speech, leading to a dynamic and interactive learning through the student's involvement on his own learning. We present an authorial environment for the creation of augmented reality applications, called A3RA, aiming at this technology democratization, incorporating it into pedagogical work, with the intention of teaching contributing. The study was based on the analysis of existing similar proposals and available tools, supporting the prototypeconstruction, still in experimentation, that includes the elaboration of environment uses situations and the exploration in physical space.
\end{abstract}

Resumo. A realidade aumentada permite que o aluno visualize uma idéia, antes apenas mentalizada a partir da fala do professor, levando para uma aprendizagem mais dinâmica e interativa por meio do envolvimento deste em sua própria aprendizagem. Apresentamos um ambiente autoral para criação de aplicações de realidade aumentada, denominado A3RA, visando a democratização dessa tecnologia, incorporando-a no trabalho pedagógico, na intenção de contribuir com o aprendizado. O estudo partiu da análise de propostas similares existentes e análise de ferramentas disponíveis, dando suporte à construção de um protótipo, ainda em experimentação, que compreende a elaboração de situações de utilização do ambiente e a exploração em campo.

\section{Introdução}

A revolução propiciada pelas TIC (Tecnologias da Informação e Comunicação) está formando uma geração de alunos que crescem usufruindo ambientes ricos em recursos multimídia, com expectativas e visão de mundo diferente das gerações anteriores. Dessa forma, exige-se da escola, novos métodos e meios de aquisição do saber, principalmente, exige-se mecanismos que apresentem oportunidades de buscas pela informação e privilegie a autonomia e a imaginação.

Nesse contexto, o processamento cada vez mais avançado dos dispositivos móveis nos possibilita o uso de uma tecnologia chamada de Realidade Aumentada (RA), que com a computação ubíqua, isso faz com que o usuário adquira autonomia e potencialize sua imaginação, fazendo-o interagir com o objeto de aprendizagem apenas ao olhar, bem como, visualizar o invisível. 
$\mathrm{O}$ que e como os alunos aprendem em ambientes de aprendizagem com realidade aumentada? Pesquisas indicaram que os sistemas e ambientes de RA podem ajudar os alunos a desenvolverem habilidades e conhecimentos que podem ser aprendidos em outros ambientes de aprendizagem aprimorados pela tecnologia, mas de uma forma mais eficaz. Sistemas de realidade aumentada são usados para apresentar situações em um formato 3D para que os alunos possam virtualmente manipular uma variedade de objetos de aprendizado e manipular as informações de forma inovadora e interativa [Wu et. al., 2012].

A realidade aumentada fornece aos alunos experiências que permitem mesclar o mundo real com o virtual, o que os leva a participar da exploração do mundo real usando objetos virtuais, como textos, vídeos, e imagens que são elementos complementares para os alunos conduzirem investigações do mundo real. A oportunidade do aluno de manipular e interagir com o real, por meio da tecnologia, vai possibilitar a visualização do mundo em torno dele, envolvendo-o em novas formas de ver questões realistas em um contexto de conexão que já é comum aos mesmos. Os objetos reais aumentados, além de criar novas formas de ver o mesmo objeto, também, ajudam os alunos a melhorar a compreensão de conceitos ou fenômenos abstratos e a partilhar os seus próprios aprendizados [Dunleavy et. al., 2009].

Embora esta tecnologia já esteja disponível e em uso, a sua chegada à escola ainda depende do trabalho de um especialista. Buscando aproximar professores e alunos nestas novas linguagens, no presente artigo apresentamos um ambiente autoral para criação de aplicações de realidade aumentada que denominamos de sistema A3RA.

O sistema A3RA é composto por um ambiente web onde o professor, a partir de um conteúdo de seu planejamento, insere objetos multimídia, configurando eventos quando tais objetos forem acionados, dessa forma organiza situações de aprendizagem inserindo roteiros e objetos em um sistema tecnologicamente estruturado em um ambiente cliente, onde os alunos vivenciam com recurso da realidade aumentada o que foi projetado pelo professor. O A3RA foi pensado de forma que haja um mínimo de esforço com a utilização da tecnologia, abstraindo a complexidade para o usuário.

O trabalho está organizado da seguinte forma: A seção 2 aborda o conceito da realidade aumentada, seu uso e técnicas; A seção 3 destaca os trabalhos relacionados; O sistema é descrito na seção 4; A seção 5 sobre o teste com o sistema e, finalmente, a seção 6 apresenta algumas considerações sobre o ambiente proposto e sinaliza os trabalhos futuros.

\section{Computação Ubíqua, Realidade Aumentada e a Affordance}

A Computação Ubíqua permite informação e suporte computacional ao usuário em qualquer espaço e tempo, de maneira invisível [Barbosa et. al., 2007], permitindo que novos conceitos e tecnologias sejam incorporados ao nosso dia a dia, o que vem facilitar a comunicação e a troca de informações. O sistema e recursos da computação podem interagir com o ser humano a todo o momento, não importando onde ele esteja, constituindo um ambiente altamente distribuído, heterogêneo, dinâmico, móvel e interativo. A informação pode ser obtida através de posicionamento de satélites e/ou através de antenas wireless. Além disso, a rápida proliferação de antenas wireless torna previsível uma crescente precisão da localização, estimulando a criação de serviços especializados (LBS, Location Based Services) [Barbosa et. al., 2007]. A possibilidade 
de aplicar as tecnologias da Computação Ubíqua para apoiar a aprendizagem abriu a área de pesquisa que é denominada Educação Ubíqua (Ubiquitous Learning). Na educação ubíqua o aprendiz tem a possibilidade de mover-se constantemente em um cenário, acessando informações e recursos necessários para construção da sua aprendizagem.

A realidade aumentada refere-se à combinação de coordenadas de componente de mídia/informação que representam objetos/mundo real(s), de forma virtual, sintética e gerada por computador em um ambiente real. Consiste na sobreposição de ambientes reais e virtuais, em tempo real, através de um dispositivo tecnológico [Kirner et. al., 2005]. Os componentes podem ser representados por modalidade visual, auditiva, tátil, olfativa [MPEG-MAR, 2017].

O termo realidade virtual é definido como "Ambiente tridimensional, gerado por computador, interativo, em que uma pessoa está imerso" [Amin e Govilkar 2015]. Há uma diferença entre o conceito de realidade virtual e a RA que pode ser explicada pelo modelo de referência MPEG-MAR (2017). Um sistema MAR (Mixedand Augmented Reality) ou um conteúdo multimídia pode ser definido de acordo com a proporção relativa do real e virtual, abrangendo a realidade física em uma extremidade, e a realidade virtual na outra extremidade, conforme figura 1 .

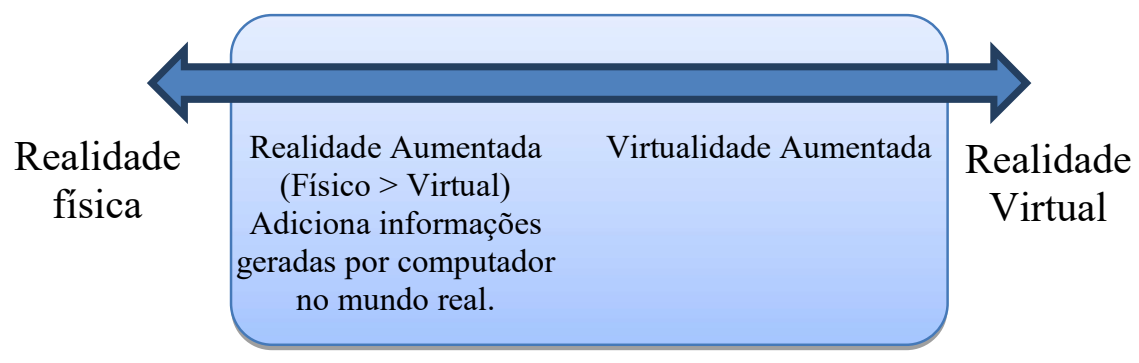

Figura 1: Continuum de realidade mista [Fraga2016].

A RA encontra-se nesse continuum mais perto do espectro do mundo real, aumentado por geração computacional. A virtualidade aumentada é a definição de sistemas que são principalmente sintéticos com algumas imagens do mundo real. Dessa forma a realidade virtual imerge o usuário completamente em um ambiente virtual em que não se pode ver o mundo real ao seu redor.

Além disso, para destacar as características da RA, temos a imersão, a ubiquidade, multiplicidade (usuário único para vários objetos) e a affordance. A dimensão da imersão é igual ao continuum realidade-virtualidade descrito pela MPEG-MAR (2017). A dimensão de ubiquidade refere-se às questões de como e onde o sistema pode ser usado, enquanto a multiplicidade envolve o grau de utilização por usuários simultaneamente, já a affordance refere-se a interação do usuário com a RA [Wu et. al., 2012].

Wu et. al. (2012) identifica características das affordances em sistemas de RA, em cinco aspectos com base em pesquisas que exploram a RA para fins educacionais. Segundo a autora, a RA poderia, tanto permitir o desenvolvimento de conteúdo de aprendizagem em perspectiva 3D, como também, a aprendizagem ubíqua, colaborativa e situada, ainda, oferece aos aprendizes os sentidos da presença, imediação, e imersão dos alunos, bem como, a capacidade de visualizar o invisível e, por último trabalha a aprendizagem 
formal e informal. Em relação ao conteúdo de aprendizagem em perspectiva tridimensional (3D), a RA pode melhorar experiências de aprendizagem usando objetos virtuais/sintéticos para os alunos interagirem, aumentando a percepção visual do sistema destino ou do ambiente. Um segundo aspecto diz respeito as affordances no contexto dos computadores portáteis em RA. Tais aspectos são devido a tecnologia prover mobilidade, conexão sem fio e tecnologia de localização, permitindo a aprendizagem ubíqua, de forma colaborativa e reforçada por simulações, jogos, modelos e objetos virtuais em ambientes reais, fornecendo ao aprendiz as affordances para a portabilidade, interatividade social, a sensibilidade de contexto, conectividade e individualidade. Em terceiro lugar oferece aos aprendizes affordances de presença, imediação, e imersão. A RA pode proporcionar um espaço que dá aos alunos a sensação de estar em um lugar com outras pessoas o que aumenta a sensação de presença e reconhecimento da comunidade deste alunos, o feedback imediato e o sentimento de onipresença, que é a impressão subjetiva de que se está participando de uma experiência realista abrangente.O quarto aspecto sobrepõe objetos ou informações virtuais em objetos físicos ou ambientes, permitindo a visualização de conceitos ou eventos difíceis de ver. Objetos reais aumentados criam novas formas de ver o mesmo objeto e têm potencial para melhorar a compreensão de conceitos ou fenômenos abstratos. O quinto e último aspecto da affordance identificada é que a RA tem o potencial para fazer a ponte entre a aprendizagem que ocorrem em contextos formais de sala de aula e informais que estão além da sala aula.

A RA, com affordance apropriada, pode ajudar os alunos a desenvolverem habilidades e conhecimentos de uma forma mais eficiente. De acordo com Dunleavy et. al. (2009) o benefício mais significativo da RA e sua affordance é a capacidade única de criar ambientes de aprendizagem híbridos imersivos que combinam objetos digitais e físicos, facilitando assim o desenvolvimento de habilidades, como pensamento crítico e resolução de problemas.

\section{TRABALHOS RELACIONADOS COM O SISTEMA PROPOSTO}

Um amplo estudo foi realizado na literatura e no mercado de sistemas que possam criar ambientes de realidade aumentada pelo usuário comum. Há muitas aplicações separadas no mercado, que trabalham somente com realidade aumentada por imagem ou geoposição e que envolvem a criação pelo autor. Há novos produtos como por exemplo o ThingWorx Studio [ThingWorx, 2017], ainda em desenvolvimento. A seguirapresentamos os dois principais trabalhos relacionados com a proposta deste artigo, o Ambiente ARIS e o fAIR-PLAY.

\subsection{Aris}

ARIS (Create location-based games and stories) é um sistema software para criar jogos para dispositivos móveis, passeios e histórias interativas. Os jogadores experimentam um mundo híbrido de personagens virtuais e mídia no espaço físico. Com o ARIS, o usuário pode construir uma história interativa, um passeio ou um jogo. Os jogadores podem completar missões, colecionar itens e conversar com personagens virtuais, enquanto exploram o mundo ao seu redor [ARIS, 2017]. Para trabalhar com a manipulação de localização o ARIS faz uso do Google Maps em seu editor.

O ambiente trabalha com QR codes, reconhecimento de imagens, geo-localização, tokens de Bluetooth e recentemente com mídias sociais. Uma de suas limitações são o 
controle de usuários e uma mudança constante de servidores A3RA. Percebemos também que o ARIS tem seu cliente somente para sistemas IOS, e por fim vimos a oportunidade de planejar um sistema que não fizesse somente jogos/passeios, mas pudesse criar qualquer tipo de projeto de RA, além de ser multiplataforma.

\section{2. fAR- PLAY}

O fAR-PLAY é um framework que permite a criação de jogos utilizando um espaço livre com o uso do GPS. O GPS permite ao ambiente suportar a localização que é executada pelos jogadores que usam dispositivos móveis ao ler códigos de barras, leitura de QR Code que são afixadas em vários espaços diferentes.

O fAR-PLAY é composto por quatro módulos: uma aplicação móvel onde os jogadores interagem com o jogo e um site que é relatado o estado do jogo, o framework do jogo que promove a lógica do jogo, o mundo virtual ferramentas para apoiar o jogo e um ambiente de criação, no qual os locais importantes do jogo podem ser especificados A3RA. Um ponto forte é que utiliza REST para comunicação entre a ferramenta autoral e a cliente [Gutierrez, et al., 2011]. Percebemos que embora o framework utilize o Layar [Layar, 2017], uma poderosa ferramenta de realidade aumentada, ele se limita a uso de QR Codes, o que limita seu uso, já que todos os frameworks atuais fazem uso de marcador com qualquer imagem. Não há atualização deste framework desde 2011 e não foi concebido para ser multiplataforma.

\section{Ambiente Proposto}

Quando integramos sala de aula e ambientes virtuais oportunizamos a entrada do mundo para dentro da escola e a escola para o mundo. Esse movimento de integração torna a sala de aula mais dinâmica e interativa o que exige uma nova relação pedagógica e uma nova competência técnica por parte do professor.

Assim, cabe ao professor planejar e organizar o caminho que os alunos precisam percorrer durante as investigações, inserindo indicações e questionamentos de apoio, mas, também, dando abertura para que estes possam acrescentar novas informações e, cabe aos alunos, com o uso dos seus celulares acessar e o conteúdo e realizarem as investigações propostas. O que concorre para uma aprendizagem mais dinâmica e interativa, envolvendo os alunos em seu próprio aprendizado, usando as vantagens da realidade aumentada em contextos reais do planejamento educacional desse professor.

A concepção e desenvolvimento do sistema A3RA, teve como ponto de partida um levantamento do estado da arte onde foram analisados as propostas existentes, veiculadas na literatura científica da área e na análises de ferramentas disponíveis para experimentações. Foram pesquisadas framework que além de atender ao requisito de realidade aumentada pudesse ser o mais aderente possível aos equipamentos móveis atuais, com possibilidade de alta performance gráfica e facilidades de implementação. Uma modelagem inicial deu suporte à construção de um protótipo funcional cuja avaliação viabilizou ajuste na modelagem e a elaboração de uma nova versão do protótipo. As etapas finais compreendem a elaboração de situações de uso e a utilização do ambiente e a exploração em campo de algumas aplicações produzidas com o ambiente. Foram utilizados vários conceitos que são exigidos para o desenvolvimento do sistema. Nesta seção apresentamos a concepção e os aspectos de implementação e uso do sistema. 


\subsection{Arquitetura do Sistema}

Conceitualmente o sistema foi pensado em possuir três módulos, sendo um ambiente de editor autoral, um ambiente executante que chamamos de cliente e um servidor de aplicação, que trabalha junto com o editor A3RA.

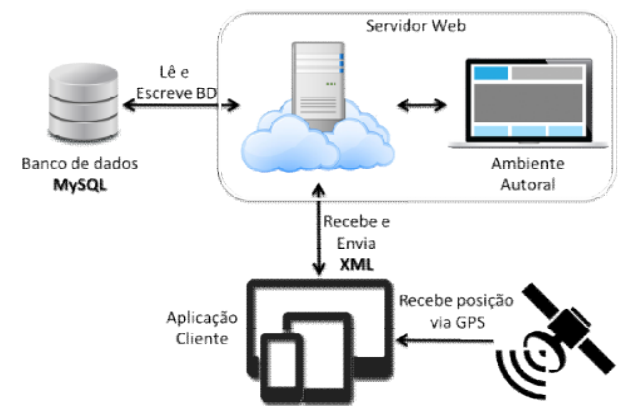

Figura 2: Arquitetura do sistema.

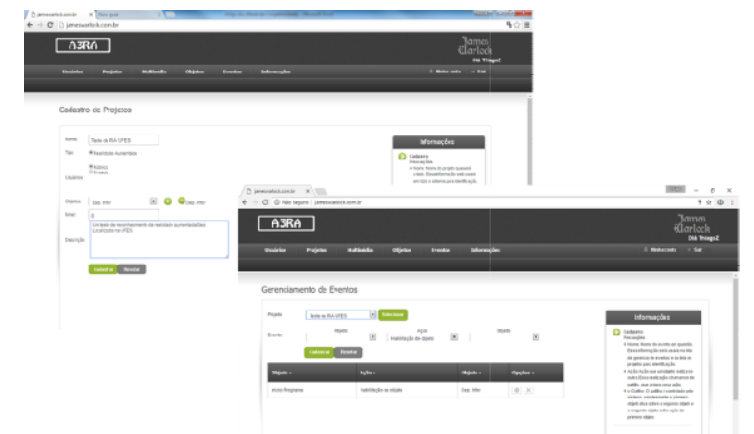

Figura 3: Tela da ferramenta autoral de criação de POl's e objetos.

\subsection{Ambiente Autoral}

O Sistema autoral tem a gestão de usuários, conteúdo multimídia, projetos de realidade aumentada e eventos. O autor tem o controle de criação de contas, tanto para perfil de administrador de projetos quanto a cadastro dos usuários, os quais poderão acessar seus projetos pelo ambiente cliente. Os projetos podem ser configurados como privados, fechados aos usuários conforme configuração do autor, e como projetos públicos, de forma que qualquer um que logar na aplicação cliente, poderá acessá-los. Para facilitar a configuração dos projetos privados foi criado o conceito de grupos, por exemplo, um professor pode agrupar alunos em salas de aula e depois se referir somente ao nome do grupo.

Para a ferramenta do autor foi planejado uma sequência de passos para que este monte seu projeto. Primeiramente o autor deve criar seu conteúdo multimídia. O A3RA trabalha com marcadores de realidade aumentada, que podem ser pontos de interesse de geoposições (POI) ou imagens a serem detectadas. O cadastro de POI's é realizado com ajuda da API do Google Maps. Na sequência é necessário criar um objeto, que nesse contexto é algo que irá interagir com o usuário. Os objetos utilizam-se desse conteúdo multimídia previamente criado e cada objeto pode conter de 1 a 4 tipos de conteúdos multimídia, sendo eles: marcador por imagem, marcador geo-localizado, imagem ou texto.

O próximo passo do autor é criar um projeto. Um projeto é o local do sistema que agrupa os objetos criados e organiza os usuários que terão acesso (ou deixa o projeto com acesso público). Esse projeto que será usado na aplicação cliente.

Por fim é necessário configurar os eventos, que são uma sequência de ações planejadas pelo autor. Por exemplo, qual objeto aparece primeiro ao usuário na aplicação cliente? Um objeto de texto pode ser exibido ao usuário quando ele entrar na aplicação cliente, e, principalmente, existe a possibilidade da criação de uma cadeia de ações, o que permite ao cliente chamar um objeto somente se outro o permitir. 
VI Congresso Brasileiro de Informática na Educação (CBIE 2017)

Anais do XXVIII Simpósio Brasileiro de Informática na Educação (SBIE 2017)

\subsection{Ambiente Cliente}

O Cliente foi concebido para ter um executante de projetos de realidade aumentada de forma genérica. Para o sistema somente existe um grande projeto de realidade aumentada, que se modela conforme for configurado no sistema autoral.

No ambiente cliente basta fazer login com o usuário, encontrar o projeto que criado pelo autor, se é privado ou público, e selecioná-lo. Os projetos serão listados conforme foi configurado o acesso pelo autor do projeto. $O$ cliente tem que ser rápido em sua utilização, então foi pensado somente nesses passos e já será possível entrar no projeto.

Nesse momento o cliente baixa as informações do sistema autoral por serviço web. Uma vez dentro do projeto carregado na aplicação cliente, o usuário pode vivenciar a aplicação. Utilizando o dispositivo móvel o usuário poderá se locomover para encontrar as posições, detectar as imagens com a filmagem da câmera em busca de objetos configurados pelo autor, para, assim poder interagir com os mesmos.

\subsection{Implementação}

O sistema autoral do A3RA está preparado para funcionar no browsers Google Chrome e Firefox. Esse requisito é devido ao programa ser o mais acessível possível e ter a codificação mais simplificada. O ambiente autoral foi desenvolvido em PHP para ficar o máximo alinhado aos códigos fornecidos pelo fabricante da tecnologia de realidade aumentada utilizada, a Vuforia. O ambiente autoral está no servidor e trabalha em RESTFul, se comunicando com o cliente por serviço web.

O ambiente cliente do A3RA foi desenvolvido em Unity3D. Escolhemos essa engine de jogos para facilitar a implantação de recursos multimídia ao projeto e a compilação para várias plataformas moveis. Embora o foco desse estudo seja em plataforma Android, com essa escolha, podemos portar para IOS ou Windows Phone, quando necessário.

A escolha da Vuforia como ferramenta de realidade aumentada foi resultado de uma pesquisa na literatura e no mercado. Nessa pesquisa concluímos que não há uma ferramenta que atenda a todos os requisitos necessários, como a realidade aumentada por detecção de imagem, e a realidade aumentada geo-localizada e que pudesse ser desenvolvida para dispositivos móveis. As ferramentas sempre atendiam 1 ou 2 requisitos somente. Devido a isso usamos o Vuforia para realidade aumentada por detecção de imagem e desenvolvemos o nosso código de realidade aumentada geoposicionada.

\section{Uma Aplicação usando o sistema Proposto}

Concebemos um roteiro que remete a um planejamento que o professor poderia realizar para o estudo de um determinado tema em uma área de conhecimento, indo além do que comumente utilizaria como recurso metodológico para o desenvolvido de suas aulas.

Tomando como exemplo um tema de história, no caso, sobre o sítio histórico da Igreja dos Reis Magos em Serra, Espírito Santo, se desenvolvido em uma sala de aula convencional, geralmente, o professor expressaria seu conhecimento enquanto os alunos seriam expectadores. Utilizaria o quadro, talvez algumas figuras e até recurso multimídia, como apoio pedagógico. 
O que propomos com a aplicação da RA, é que o aluno saia da posição de expectador passivo e passe a vivenciar e interagir com o ambiente, por meio de observações e análise do espaço relacionado ao conteúdo, o que concorre para uma aula ativa e dinâmica, onde o papel do professor passa de repassador de informações para orientador e os alunos, com o apoio do professor, construiriam o seu próprio aprendizado.

O Sistema proposto, apresenta o assunto a ser explorado como se o aluno tivesse uma "lente" que o faz ver o passado, apresentando itens que não existem mais, ou sofreram grandes modificações. Visualizando a construção os alunos poderão interagir com o passado, com informações relevantes para seu aprendizado, vivenciando situações que seria impossível em uma sala de aula convencional e oportunizando a motivação e colaboração entre os alunos com base em suas descobertas, contribuindo para a motivação e a aprendizagem dos mesmos.

\subsection{Concepção}

Apresentaremos o planejamento e a execução de uma aplicação criada no ambiente autoral de nosso protótipo.

Escolhemos um sítio histórico local, simulando uma curta aula de história ambientada no espaço da Igreja dos Reis Magos em Serra, Espírito Santo. A base do roteiro é a história do local e suas lendas, apresentadas pelos guias turísticos que ficam na igreja.

A igreja foi tombada pelo IPHAN (Instituto do Patrimônio Histórico e Artístico Nacional) no ano de 1943, localiza-se no bairro de Nova Almeida, numa região onde existia um núcleo de catequese indígena entre os séculos XVI e XVIII. A igreja fica a 40 metros acima do nível do mar, de onde é possível ter uma visão privilegiada da orla de Nova Almeida. Além de igreja, podemos considerar o local uma espécie de museu e sítio arqueológico.

Foi criado então um projeto no editor autoral de passeio na Igreja dos Reis Magos, com o seguinte planejamento:

1) Estando no sitio arqueológico, ao logar no aplicativo cliente, o sistema mostrará a localização da igreja com uma imagem mapmaker no local da cruz, na praça dos coqueiros, que fica em frente da igreja (figura 4);

2) A seguir, uma informação será exibida na tela do cliente, explicando que "a igreja foi fundada por padres jesuítas por volta de 1580 e era chamada inicialmente de Aldeia dos Reis Magos. A primeira capela foi inaugurada no dia 06 de janeiro de 1557 pelo padre Brás Lourenço". Visualize agora a igreja. Nesse momento fica habilitado o objeto igreja e na geoposição da igreja real será exibida uma casa de pau a pique em 3D (figura 5);

3) Ao filmar partes da igreja do lado de fora é possível que apareça um pássaro em $3 \mathrm{D}$ e um informativo de uma das lendas. No século 16 a igreja se tornou uma cadeia municipal, e existia uma lenda de que um índio que foi preso fugiu usando magia se transformando em um pássaro. Nesse momento houve um questionamento sobre o que foi visto;

4) Por fim, quando o usuário chegar no mirante, em que é possível ter a vista do local, será exibida uma informação de que esta é a vista que as pessoas viam na época e que continuam vendo atualmente. 


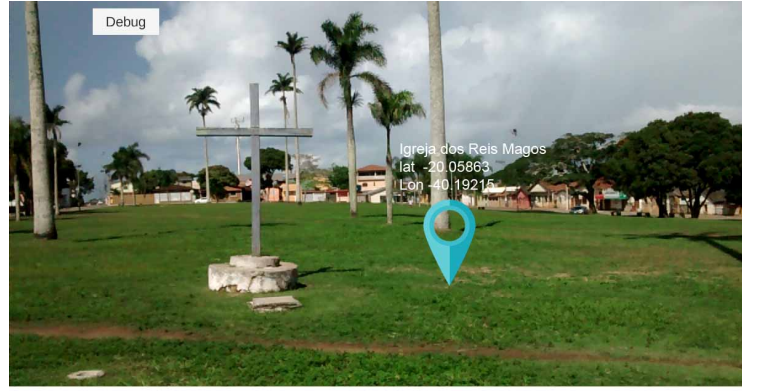

Menu Principal Cackstra POI Teste

Figura 4: Tela do cliente apresentando um ponto de interesse (POI).

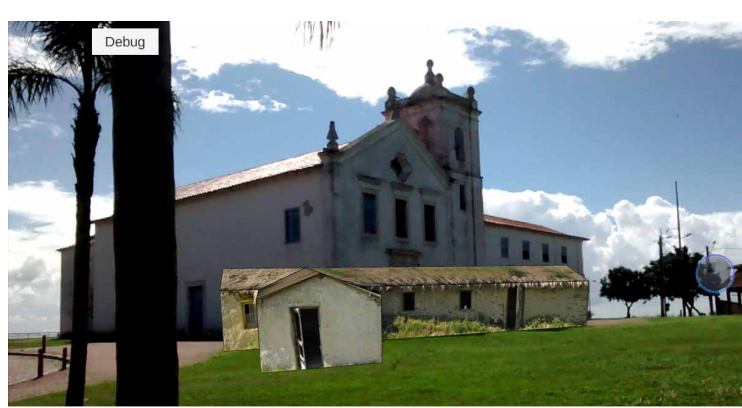

\begin{tabular}{ll|l} 
Menu Principal & Cadastra POI Teste \\
\hline
\end{tabular}

Figura 5: Tela do cliente apresentando uma imagem 3D em uma geo-posição.

$\mathrm{Na}$ situação 1 foi usado somente realidade aumentada com geoposição e exibido um texto informativo; já na situação 2 foi usado realidade aumentada com geoposição e no local e foi exibida uma imagem 3D dispobilizada gratuitamente na 3D Warehouse do Google Sketchup [3D Warehouse 2017]; na situação 3 usamos o feedback do usuário e a realidade aumentada por detecção de imagens, onde o marcador é a janela da igreja, e nela exibimos o pássaro e um informativo; por ultimo, a situação 4 é usado a mesma técnica da situação 1 .

\subsection{Avaliando o protótipo}

Para o teste foi utilizado um aparelho Android na versão 5.0 (api 21), quad-core $1.2 \mathrm{GHz}$ e $1 \mathrm{~Gb}$ RAM. A aplicação rodou bem durante todo o teste. O sistema cliente necessita obrigatoriamente de estar em rede, utilizamos a rede $3 \mathrm{G}$. Durante o teste quando houve lentidão da rede o aparelho cliente também teve lentidão, principalmente quando detectou a imagem da janela, pois faz uma verificação direto do serviço web da Vuforia.

Observamos a existência de uma limitação que existe no uso do A3RA, referente ao ambiente cliente rodar em celulares comuns, que possuem sensores de baixa qualidade (GPS, Câmera, acelerômetro...), a aplicação cliente gera pequenas inconsistências. O GPS tem em média um erro de 12 metros, chegando a falhas de 50 metros. Por causa dessa limitação, o sistema A3RA, deve ser utilizado em grandes áreas para vivenciar os projetos. Fato, este, já considerado no planejamento do protótipo. Essa limitação não impede que o sistema rode e as informações e imagens sejam exploradas conforme o planejamento.

É possível afirmar, por meio deste experimento, que o A3RA tem como contribuir com o cenário educacional, ao transformar uma sala de aula convencional em uma aula de campo, onde o aluno vivencia o espaço do conteúdo trabalhado, fora dos muros da sala de aula.

\section{Considerações Finais}

A grande disseminação e facilidade no uso dos dispositivos móveis contribuiu para o uso de Realidade Aumentada (RA) com a computação ubíqua. Essa condição oportuniza ao aluno de manipular e interagir com o real, por meio da tecnologia, possibilitando a visualização do mundo em torno dele, envolvendo-o em novas formas de ver questões realistas em um contexto de conexão que já é comum aos mesmos. Essas experiências 
VI Congresso Brasileiro de Informática na Educação (CBIE 2017)

Anais do XXVIII Simpósio Brasileiro de Informática na Educação (SBIE 2017)

levarão aos mesmos a mesclar o mundo real com o virtual, possibilitando exploração deste mundo real.

Neste trabalho apresentamos uma proposta com base em tecnologias e conceitos atuais, além de trazer uma nova abordagem de multiplataforma e acessibilidade dos dados do servidor.

Como proposição para trabalhos futuros será aprimorar o upload de models 3D no sistema, configurando melhor como ele será exibido na aplicação cliente; A criação de um ambiente de acompanhamento para que o professor gere todos os relatórios necessários, como também, melhor a flexibilidade dos cadastros no sistema e seu controle dados. Será necessário, ainda, que seja melhorado o cadastro de geoposição no sistema autoral a ponto de facilitar a modificação de pontos já criados, movendo-os no mapa, clicando e arrastando com o mouse e por fim, planejar quais serão as próximas plataformas móveis que serão contempladas no sistema.

Em relação á aplicabilidade nas escolas, buscaremos contato com professores, faremos oficinas gratuitas para disseminar o uso deste ambiente e divulgar a cultura das aplicações desta natureza, com vistas à validação do sistema.

\section{Referencias}

Wu, Hsin-Kai; Lee, Silvia Wen-Yu; Chang, Hsin-Yi; Liang, Jyh-Chong (2012), "Current status, opportunities and challenges of augmented reality in education", Computers \& Education.

Dunleavy, Matt; Dede, Chris; Mitchell, Rebecca (2009), Affordances and Limitations of Immersive Participatory Augmented Reality Simulations for Teaching and Learning, Journal of Science Education and Technology.

Barbosa, Jorge; Hahn, Rodrigo; Rabello, Solon; Pinto, Sérgio Crespo C. S.; Barbosa, Débora Nice Ferrari (2007), "Computação Móvel e Ubíqua no Contexto de uma Graduação de Referência", Revista Brasileira de Informática na Educação Volume 15 - Número 3.

Kirner, Claudio e Zorzal, Ezequiel Roberto (2005), "Aplicações Educacionais em Ambientes Colaborativos com Realidade Aumentada", XVI Simpósio Brasileiro de Informática na Educação - SBIE - UFJF.

MPEG-MAR, ISO/IEC 18039, "MixedandAugmented Reality ReferenceModel" $<$ http://mpeg.chiariglione.org/standards/mpeg-mar>, acessado em maio 2017.

ThingWorx Studio, <https://www.thingworx.com/platforms/thingworx-studio/pilot/>, acessado em maio 2017.

ARIS <https://fielddaylab.org/make/aris/>, acessado em maio 2017.

Gutierrez, L.; Nikolaidis, I.; Stroulia, E.; Gouglas, S.; Rockwell, G.; Boechler, P.; Carbonaro, M.; King, S. (2011) "fAR-PLAY: A framework to develop Augmented/Alternate Reality Games", IEEE Workshop on Pervasive Collaboration and Social Networking.

Layar $<$ https://www.layar.com/>, acessado em maio 2017.

Google 3D Warehouse $<$ https://3dwarehouse.sketchup.com/?hl=pt-BR $>$, acessado em maio 2017. 